Artigo / Article

\title{
Impacto da recusa de candidatos à doação de sangue devido aos critérios para prevenção da doença de Creutzfeldt-Jacob e sua variante
}

\author{
Impact of deferral criteria on prevention of Creutzfeldt-Jakob disease and its variant among blood \\ donors in São Paulo, Brazil
}

Cesar Almeida Neto ${ }^{1,2}$

Alfredo Mendrone Júnior ${ }^{1,2}$

Anésio Vieira

Pedro E. Dorlhiac-Llacer ${ }^{1,2}$

Dalton AF Chamone ${ }^{1,2}$
A doença de Creutzfeldt-Jacob (CJD) e sua variante (vCJD) são doenças neurológicas fatais, degenerativas, com longo periodo de incubação. Recentemente, um possível caso de transmissão de vCJD por transfusão sangüínea foi descrito. A partir de 2001 adotamos critérios para recusa de candidatos baseados em dados da literatura, Portarias e Resoluções do Ministério da Saúde. Avaliamos retrospectivamente o porcentual de recusas anuais na triagem clínica de 1.015 .587 candidatos à doação, referentes aos critérios relacionados a CJD e vCJD, de janeiro de 2001 a dezembro de 2004. Este porcentual correspondeu a 0,0049\% em 2001, 0,0051\% em 2002, 0,092\% em 2003 e 0,098\% em 2004, representando recusa definitiva de 726 (0,068\%) candidatos. A causa mais freqüente de recusa por CJD ou vCJD foi permanência no Reino Unido > 6 meses (259 recusas) seguido de transplante de córnea (203 recusas) e uso de hormônio do crescimento de origem humana (151 recusas). Embora o número de recusas relacionados a CJD e sua variante seja baixo em nossa Instituição, observamos um aumento gradativo anual, que provavelmente relaciona-se à adição de novos critérios. Diferentemente de outros países, estas recusas não afetaram nosso estoque de sangue e componentes. Com os dados obtidos, consideramos que, na nossa população, podemos manter o "Princípio da Precaução" sem causar prejuizo no fornecimento de sangue e componentes aos nossos pacientes. Rev. bras. hematol. hemoter. 2005; 27(2):106-109.

Palavras-chave: Doadores de sangue; Doença de Creutzfeldt-Jacob; prions.

\section{Introdução}

Na década de 1990, conseqüentemente à epidemia da Encefalopatia Bovina Espongiforme (EBE) no Reino Unido, houve grande interesse da comunidade internacional em estudar a possibilidade de contaminação dos estoques de sangue com príons patogênicos. ${ }^{1}$

As doenças causadas por príons em humanos incluem a Doença de Creutzfeldt-Jacob, de origem idiopática e de formas hereditárias, associadas com mutações do gene da proteína do príon; a variante da doença de Creutzfeldt-Jacob, que parece estar relacionada ao agente da EBE; e os casos iatrogênicos transmitidos via hormônios humanos de origem hipofisária, enxertos de dura-máter, transplantes de córnea e procedimentos neurocirúrgicos. $^{2}$

Em 1996, Will et al ${ }^{3}$ relataram dez casos de CJD no Reino Unido, que ocorreram em pacientes relativamente jovens, cujo curso clínico era diferente do até então tipicamente encontrado. Este grupo propôs que estes casos estavam relacionados à EBE, baseados em achados epidemiológicos e neuropatológicos, e adotaram o termo nova variante da CJD, ou nvCJD, mais tarde denominada variante da CJD (vCJD).

${ }^{l}$ Fundação Pró-Sangue - Hemocentro de São Paulo.

${ }^{2}$ Departamento de Hematologia da Faculdade de Medicina da Universidade de São Paulo.

Correspondência para: César Almeida Neto

Fundação Pró-Sangue Hemocentro de São Paulo - Ambulatórios de Doadores

Av. Dr. Enéas Carvalho de Aguiar, $1551^{\circ}$ andar - Cerqueira Cesar

05403-000 - São Paulo-SP

E-mail: cesarnt@uol.com.br 
Com a descoberta de príons patogênicos em tecidos linfóides de pacientes com vCJD postulou-se a possibilidade de transmisssão deste agente por vias periféricas, ${ }^{4}$ tal como transfusões sangüíneas. Em dezembro de 2004, o Ministro da Saúde inglês, John Reid, relatou ao Parlamento que um paciente havia falecido com diagnóstico confirmado de vCJD no dia 17 daquele mês. Este paciente havia recebido em 1996 transfusão de sangue de um doador sadio, que, em 1999, falecera de vCJD. Reid afirmou que a doença poderia ter sido transmitida do doador para o receptor via transfusão sangüínea, porém a possibilidade de ambos terem se alimentado de carne infectada por EBE também deveria ser cogitada. ${ }^{5}$ Tendo em vista, até o momento, não haver testes de triagem fidedignos para a detecção de indivíduos infectados pelos príons patogênicos, ${ }^{6}$ vários países, dentre eles o Brasil, adotaram medidas de recusa "geográfica" de candidatos à doação de sangue de acordo com o "Princípio da Precaução."7

A recusa de candidatos a doação devido a fatores possivelmente correlacionados à transmissão de príons patogênicos é polêmica, causando sentimentos de frustração nos candidatos, aumento no número de recusas e eficácia questionável. Em nosso meio não há estimativas do impacto da recusa de doadores nos grandes hemocentros.

O objetivo deste estudo é avaliar o impacto dos critérios de recusa para CJD e vCJD de candidatos à doação de sangue na nossa instituição, desde a implantação destes, em janeiro de 2001 até dezembro de 2004, e discutir as medidas que podem ser adotadas tanto na orientação dos candidatos quanto na hemovigilância.

\section{Casuística e Método}

Foram avaliadas todas as triagens clínicas dos candidatos à doação que compareceram na Fundação Pró-Sangue - Hemocentro de São Paulo entre janeiro de 2001 e dezembro de 2004, calculando-se o impacto dos critérios de recusa para CJD e vCJD nesta população.

A partir de 2001 adotamos critérios para recusa de candidatos baseados em dados da literatura, Portarias e Resoluções do Ministério da Saúde.

Em 2001, os critérios adotados para exclusão de candidatos foram história familiar de CJD, uso de hormônio dos crescimento de origem humana e transplante de córnea. ${ }^{8}$ Em 2002 passamos também a recusar candidatos que estiveram de forma cumulativa ou não por mais de seis meses no Reino Unido entre 1980 e $1996 .{ }^{9} \mathrm{Em} 2003$, com a RDC n ${ }^{\circ}$ $34.3,{ }^{10}$ candidatos que estiveram de forma cumulativa ou não por mais de seis meses no Reino Unido ou República da Irlanda a partir de 1980 foram recusados. Nesta ocasião, baseados nos critérios do FDA e AABB,${ }^{11}$ recusamos ainda candidatos que estiveram por mais de dez anos cumulativos na França e Portugal desde 1980. Com a RDC n ${ }^{\circ} 15.3,{ }^{12}$ estes critérios foram revistos e a recusa passou a ser de doadores que estiveram por seis meses ou mais no Reino Unido, entre 1980 e 1996, e por mais de dez anos na França, Portugal ou República da Irlanda desde 1980, mantendo-se as restrições para aqueles que receberam hormônio do crescimento ou outros medicamentos de origem hipofisária, transplante de córnea ou implante de material biológico à base de dura-máter, história familiar de EBE.

Comparamos ainda a porcentagem de recusas na triagem clínica dos candidatos a doação nos quatro anos precedentes à implantação destes critérios com as ocorridas quatro anos após.

\section{Resultados}

Em 2001, 2002, 2003 e 2004, o porcentual de recusas de doadores de acordo com os critérios estabelecidos corresponderam a $0,049 \%, 0,051 \%, 0,092 \%$ e $0,098 \%$ respectivamente. Isto representou recusa definitiva de 726 candidatos num universo de 1.015 .587 doadores $(0,068 \%)$. A causa mais freqüente de recusa por CJD ou vCJD foi permanência no Reino Unido $>6$ meses (259 recusas) seguido de transplante de córnea (203 recusas) e uso de hormônio do crescimento de origem humana (151 recusas). Os demais critérios somaram apenas 113 recusas.

Observamos também que, no decorrer do tempo, houve um discreto aumento na quantidade de doadores recusados (Tabela 1).

Quando comparamos as taxas de recusa na triagem clínica pelos mais diversos motivos nos quatro anos anteriores à implantação dos critérios de recusa para CJD e vCJD com aquelas ocorridas quatro anos após, notamos que, entre 1997 e 2000, ocorreram 234.302 recusas para 1.197 .036 candidatos $(19,5 \%)$ e entre 2001 e $2004,192.259$ recusas para 1.015 .587 candidatos $(18,9 \%)$ (Tabela 2$)$.

\section{Discussão}

Há um pequeno número de casos de vCJD descritos na literatura médica. Estes ocorreram em uma região geográfica limitada. Até o momento, foram descritos 146 casos de vCJD no Reino Unido, ${ }^{13}$ seis casos na França e casos esporádicos na República da Irlanda, Itália, EUA, Canadá e Japão. Em todos os casos, exceto os descritos na França e na Itália, os doentes residiram no Reino Unido, e, em todos os casos testados, apresentaram homozigose para metionina no códon 129 do gene PRNP. Devido a esta peculiaridade da doença, ausência de um teste para detectar portadores da infecção por príons patogênicos e a potencial transmissibilidade da doença por transfusões sangüíneas, foram criados critérios geográficos para a recusa de doadores. Estes critérios devem representar um balanço entre a precaução e a disponibilidade de doadores de sangue, ou seja, não é factível criar critérios de segurança que diminuam drasticamente os estoques de sangue. 


\begin{tabular}{|c|c|c|c|c|c|c|c|c|c|c|}
\hline \multicolumn{11}{|c|}{$\begin{array}{l}\text { Tabela } 1 \\
\text { Total de candidatos e porcentagem de recusa devido aos critérios para CJD e vCJD na FPS/HSP entre } 2001 \text { e } 2004\end{array}$} \\
\hline & \multicolumn{2}{|c|}{2001} & \multicolumn{2}{|c|}{2002} & \multicolumn{2}{|c|}{2003} & \multicolumn{2}{|c|}{2004} & \multicolumn{2}{|c|}{ 2001-2004 } \\
\hline & \multicolumn{2}{|c|}{$n=292184$} & \multicolumn{2}{|c|}{$n=267621$} & \multicolumn{2}{|c|}{$n=230786$} & \multicolumn{2}{|c|}{224996} & \multicolumn{2}{|c|}{$n=1.015 .587$} \\
\hline \multirow[t]{2}{*}{ Critérios de recusa } & \multicolumn{2}{|c|}{ critério CJD/vCJD } & \multicolumn{2}{|c|}{ critério CJD/vCJD } & \multicolumn{2}{|c|}{ critério CJD/vCJD } & \multicolumn{2}{|c|}{ critério CJD/vCJD } & \multicolumn{2}{|c|}{ critério CJD/vCJL } \\
\hline & $n D^{*}$ & $\%$ DR & n DR & $\%$ DR & n DR & $\%$ DR & $\mathrm{nDR}$ & $\%$ DR & n DR & $\% \mathrm{DR}$ \\
\hline Familiar com CJD & 56 & 0,019 & 2 & $<0,000$ & 0 & 0 & 3 & 0,001 & 61 & 0,006 \\
\hline Horm.crescimento & 58 & 0,019 & 32 & 0,011 & 33 & 0,014 & 28 & 0,012 & 151 & 0,014 \\
\hline Tx córnea & 33 & 0,011 & 38 & 0,014 & 67 & 0,029 & 65 & 0,028 & 203 & 0,019 \\
\hline$>10$ anos França & & & & & 6 & 0,002 & 9 & 0,004 & 15 & 0,001 \\
\hline$>10$ anos Portugal & & & & & 15 & 0,026 & 8 & 0,003 & 23 & 0,002 \\
\hline > 10 anos Rep. Irlanda & & & & & 4 & 0,001 & 10 & 0,004 & 14 & 0,001 \\
\hline$>6$ meses Reino Unido & & & 66 & 0,024 & 88 & 0,038 & 105 & 0,046 & 259 & 0,025 \\
\hline Total & 147 & 0,049 & 138 & 0,051 & 213 & 0,092 & 228 & 0,098 & 726 & 0,068 \\
\hline
\end{tabular}

\begin{tabular}{cccc}
\multicolumn{5}{c}{ Tabela 2} \\
\multicolumn{4}{c}{ Candidatos e porcentagem de recusa na triagem clínica } \\
da FPS/HSP entre & 1997 e 2004 \\
\hline Ano & $\mathrm{n}$ Candidatos & $\mathrm{n} \mathrm{DR}$ * & $\%$ \\
\hline 1997 & 308.856 & 68.311 & 22,12 \\
1998 & 313.751 & 63.689 & 20,30 \\
1999 & 293.965 & 54.209 & 18,44 \\
2000 & 280.464 & 48.093 & 17,15 \\
2001 & 292.184 & 54.761 & 18,74 \\
2002 & 267.621 & 49.214 & 18,39 \\
2003 & 230.786 & 48.719 & 21,11 \\
2004 & 224.996 & 44.565 & 19,81 \\
\hline Total & 2.212 .623 & 431.561 & 19,50 \\
\hline *DR = doador recusado & & \\
\hline
\end{tabular}

O FDA estimou, com os critérios adotados nos EUA, uma perda de $5 \%$ dos doadores, e a Cruz Vermelha NorteAmericana, com critérios mais restritivos ainda, uma perda de $8 \%$ a $9 \%$ dos doadores. ${ }^{14}$ Murphy et al,${ }^{15}$ em publicação recente, estimaram a perda de doadores devido aos critérios de recusa geográfica para $\mathrm{vCJD}$ através de questionários anônimos. Houve uma variação de $1 \%$ ou menos até $13 \%$ de recusas, sendo o principal motivo naquele estudo o Serviço Militar em uma base na Europa.

No nosso serviço, as recusas devidas aos critérios para CJD e vCJD foram baixas provavelmente em razão das características inerentes da nossa população, e, além do mais, não causaram impacto quanto à taxa global de recusas de candidatos à doação após sua implantação. Embora a cidade de São Paulo tenha uma renda per capita elevada em relação ao restante do país, provavelmente são poucas as pessoas que viajam para o exterior, que têm acesso a tratamentos de alto custo, tal como hormônio de crescimento humano, ou tenham sido submetidas a transplante de córnea, em nosso meio.

Com o decorrer dos anos houve uma gradual tendência ao aumento de recusas devido a estes critérios, provavelmente relacionada à adição de novos critérios de recusa. Ao observarmos a porcentagem de recusas por contato familiar com doente com CJD, uso de hormônio do crescimento de origem humana e transplante de córnea, ou seja, os primeiros critérios adotados em 2001, notamos que na média, não houve grande aumento na recusa de candidatos.

Por outro lado, é importante que os bancos de sangue e hemocentros tenham condutas preestabelecidas para a orientação destes doadores. No nosso caso, 726 candidatos foram recusados em definitivo devido às normas oficiais. Acreditamos que os candidatos à doação deveriam ser recusados por tempo indeterminado, pois, aparentemente, a epidemia de vCJD está declinando no Reino Unido, embora possa haver casos desta doença com período de incubação mais prolongado, e há a expectativa de desenvolvimento de um teste capaz de identificar prováveis portadores dos príons patogênico. ${ }^{16}$ Além dos mais, estes critérios não são estáticos e, como observamos, podem sofrer modificações conforme aumente o conhecimento da epidemiologia e fisiopatologia da vCJD.

Quanto aos receptores de sangue de candidatos que anteriormente foram considerados aptos, e ao retornarem para nova doação são recusados devido aos critérios para CJD ou vCJD, diferentemente do Reino Unido, somos da opinião que os receptores não devem ser notificados. Porém, os serviços de hemoterapia devem manter uma listagem que permita a rastreabilidade destes doadores e receptores. 
Caso houver algum caso de vCJD descrito no futuro em nosso país, seria importante verificar se este doente era ou não doador de sangue. Em caso positivo deveria instalar-se retrovilância dos receptores.

Concluindo, embora o número de recusas relacionados aos critérios para CJD e vCJD seja baixo em nossa instituição, observamos um aumento gradativo anual que provavelmente se relaciona à adição de novos critérios. Os serviços de hemoterapia devem manter em seus procedimentos condutas referentes à orientação de doadores recusados e rastreabilidade de receptores de transfusões. Diferentemente de outros países, estas recusas não afetaram nosso estoque de sangue e componentes. O "Princípio da Precaução" estabelece que uma ação deve ser tomada na ausência de dados seguros quando as conseqüências da falta de ação podem ser catastróficas. Consideremos que, na nossa população, atualmente, podemos manter o "Princípio da Precaução" sem prejudicar o fornecimento de sangue e componentes aos nossos pacientes.

\begin{abstract}
Creutzfeldt-Jakob disease (CJD) and its variant (vCJD) are degenerative and fatal neurological diseases with a long incubation period. Recent studies have described a potential association between $v C J D$ and blood transmission. The aim of this study is to evaluate the impact of the deferral criteria for CJD and $v C J D$ in our institution since their implementation in 2001. Based on scientific data, we have adopted deferral criteria for the prevention of CJD and $v C J D$ through blood transfusion since 2001. Blood donors are deferred if they spent more than 6 months cumulatively in the United Kingdom from January 1, 1980 through December 31, 1996, 10 years cumulatively in France, Portugal, or the Irish Republic since 1980, received human pituitary-derived growth hormone or corneal transplants or had one or more blood relatives with familial CJD. The deferral rate concerning these criteria was retrospectively evaluated for all blood donation candidates from January 2001 to December 2004. A total of 1,015,587 pre-donation interviews were evaluated. The deferral rate for CJD and $v C J D$ was $0.049 \%$ in 2001, 0.051\% in 2002, 0.092\% in 2003 and 0.098\% in 2004, denoting a permanent deferral of $726(0.068 \%)$ blood donors. The most frequent deferral reasons for CJD and $v C J D$ was spending more than 6 months cumulatively in the United Kingdom from 1980 to $1996(0.025 \%)$ followed by corneal transplants (0.019\%) and use of pituitary-derived human growth hormone $(0.014 \%)$. The number of deferrals related to CJD and its variant was very low at our center. It is feasible to support the "Precautionary Principle" for CJD and $v C J D$ at our center without jeopardizing blood supply. Rev. bras. hematol. hemoter. 2005;27(2):106-109.
\end{abstract}

Key words: Blood donors; Creutzfeldt-Jakob disease; prions.

\section{Agradecimentos}

Agradecemos ao Sr. Mário Santos da Cunha, do Departamento de Sistemas da Fundação Pró-Sangue, pelo seu valioso trabalho na obtenção e compilação dos dados deste artigo.

\section{Referências Bibliográficas}

1. Murphy MF. New variant Creutzfeldt-Jakob Disease (nvCJD): the risk of transmission by blood transfusion and potential benefit of leukocyte-reduction of blood components. Transfusion Medicine Reviews 199;13:75-83.

2. Llewwelyn CA, Hewitt PE, Knight RSG, et al. Possible transmission of variant Creutzfeldt-Jakob disease by blood transfusion. Lancet 2004;363:417-21.

3. Will RG, Ironside JW, Zeidler M, et al. A new variant of CreutzfeldtJakob disease in the UK. Lancet 1996;347:921-5.

4. Hill AF, Butterworth RJ, Joiner S, et al. Investigation of variant Creutzfeldt-Jakob diseases with tonsil biopsy samples. Lancet 1999; 253:183-9.

5. Pincock. Patient's death from vCJD may be linked to blood transfusion. Lancet 2004;363:43.

6. Völkel D, Zimmermann K, Zerr I, et al. Immunochemical determination of cellular prion protein in plasma from healthy subjects and patients with sporadic CJD or other neurologic diseases. Transfusion 2001;41:441-8.

7. Wilson K, Wilson M, Hérbert PC, Graham I. The application of the Precautionary Principle to the blood system: the Canadian Blood System's vCJD Donor Deferral Policy. Transfusion Medicine Reviews 2003;17:89-94.

8. American Association of Blood Banks, Technical Manual, 12th edition, p. 92-4. Bethesda, Maryland, 1996.

9. EUA. Food and Drug Administration. Transmissible Spongiform Encephalopathyies. Advisory Committee Meeting em $1^{\circ}$ de junho de 2000.

10. BRASIL. Agência Nacional de Vigilância Sanitária. Resolução RDC $\mathrm{n}^{\circ} 343$, de 13 de dezembro de 2002.

11. EUA. Food and Drug Administration. Summary and Rationale for Draft Guidance. "Revised Preventive Measures to Reduce a Possible Risk of Transmission of CJD and vCJD by blood and blood products" de 27 de agosto de 2001.

12. BRASIL. Agência Nacional de Vigilância Sanitária. Resolução RDC $\mathrm{n}^{\circ} 153$, de 14 de junho de 2004.

13. The National Creutzfeldt Disease Surveillance Unit. Eleventh Annual Report. http://www.cjd.ed.ac.uk/eleventh/rep2002.htm

14. Us Food and Drug Administration. Guidance for industry. Revised preventive measures to reduce the possible risk of transmission of Creutzfeldt-Jakob disease (CJD) and variant Creutzfeldt-Jakob Disease (vCJD) by blood and blood products. January, 2002. http:/ /www.fda.gov/cber/gdlns/cjdvcjd.pdf

15. Murphy EL, David Connor J, McEvoy P, et al. Estimating blood donor loss due to the variant CJD travel deferral. Transfusion 2004;44:645-50.

16. Goodnough LT, Hewitt PE, Silliman CC. Joint ASH and AABB Educational Session. Hematology 2004, American Society of Hematology 457-72.

Avaliação: Editor e dois revisores externos.

Conflito de interesse: não declarado

Recebido: 06/05/2005

Aceito após modificações: 16/06/2005 